\title{
An Innovative Concept of High-Dose-Rate (HDR) Intracavitary Brachytherapy with an Intrauterine Inflatable Balloon for Endometrial Carcinoma
}

\author{
Abhilasha Patel*, Shannon Cummins, Join Y. Luh, Tony Eng \\ University of Texas Health Science Center at San Antonio (UTHSCSA), San Antonio, TX, USA \\ Email: ${ }^{*}$ ajpatel246@hotmail.com
}

Received 13 June 2014; revised 10 July 2014; accepted 5 August 2014

Copyright (C) 2014 by authors and Scientific Research Publishing Inc.

This work is licensed under the Creative Commons Attribution International License (CC BY).

http://creativecommons.org/licenses/by/4.0/

(c) (i) Open Access

\begin{abstract}
Purpose: Curative radiation therapy is an established treatment option for non-surgical patients with early-stage endometrial carcinoma. Dosimetric analyses were performed using a single tandem, double tandem, Heyman capsules, and an inflatable intrauterine balloon to assess the dose homogeneity and conformality in the definitive treatment of inoperable endometrial cancer. Methods and Materials: Patients' informed-consent was obtained. Dosimetric analyses were performed using four different after-loading applicators to assess the dose homogeneity and conformality of isodose to the three-dimensional (3-D) shape of the target volume (uterus) based on CT data in four patients $(n=4)$. The single tandem and double tandems were standard Fletcher-type (Nucletron Corporation, Columbia, MD). Heyman capsules were the disposable after-loading type (Radiation Products Design, Inc., Albertville, MN). The inflatable balloon with a central bi-lumen catheter was the Mammo Site Radiation Therapy System (Proxima Therapeutics, Alpharetta, GA) that is currently used for local breast brachytherapy. Treatment planning and dosimetric analyses for all four techniques were done with HDR PLATO Brachytherapy (v14.2.3) Software (Nucletron Corporation). Results: The average dose gradient within the target (uterine wall) is highest with the tandem methods, followed by Heyman capsules. The intrauterine balloon method showed the least dose gradient across the uterine wall. The corresponding average homogeneity indices were 3.81, 3.83, 2.97, 2.50 for single tandem, double tandem, Heyman capsules, and intrauterine balloon respectively. Conclusions: The intra-uterine inflatable balloon appears to have the best overall dosimetric advantages for the treatment of the uterine wall. Furthermore, the potential ease of use, shorter time of applicator placement, and better patient comfort warrant further investigation and subsequent clinical implementation.
\end{abstract}

*Corresponding author.

How to cite this paper: Patel, A., Cummins, S., Luh, J.Y. and Eng, T. (2014) An Innovative Concept of High-Dose-Rate (HDR) Intracavitary Brachytherapy with an Intrauterine Inflatable Balloon for Endometrial Carcinoma. Journal of Cancer Therapy, 5, 878-883. http://dx.doi.org/10.4236/jct.2014.59095 


\section{Keywords}

\section{Intracavitary Balloon, Brachytherapy, High-Dose-Rate, Endometrial Cancer}

\section{Introduction}

Endometrial cancer is the most common gynecologic malignancy in the United States and is the fourth most common cancer in women. The estimated incidence for US women in 2012 is 47,130 new cases [1]. Standard of care is treatment with surgical resection which includes a total abdominal hysterectomy with bilateral salpingooopherectomy in addition to pelvic lymph node sampling depending on the stage of the disease. Some high risk patients require adjuvant treatment with radiation and/or chemotherapy, or both, based on pathologic findings. In patients who are deemed to be inoperable secondary to medical comorbidities, curative radiation therapy is an established treatment option for endometrial carcinoma. Inoperable cases make up less than $5 \%$ of endometrial cancers. Some series have reported that treatment with definitive radiation results in inferior outcomes compared to definitive surgery centered primarily on the fact that radiation treatments are delivered based on clinical staging compared to surgery which is based on pathologic staging. However, multiple series have also shown that overall local control rate of $75 \%$ (range $60 \%$ - 85\%) have been achieved with disease-specific survival of approximately $65 \%(50 \%-85 \%)$ for patients with early-stage disease treated with definitive radiation [2]-[11]. These results approach those of surgical data.

Brachytherapy is an essential part of the treatment course whether it follows external treatments or is given alone following surgery. In patients who receive definitive treatment without surgery, it is an essential component of the treatment course to provide high doses to clinical disease while at the same time allowing for rapid dose fall-off to spare critical structures in the pelvis from unnecessary radiation dose. The clinical target volume (CTV) for unresectable early-stage patients typically includes the entire uterine body and the adjacent part of the cervix with the entire thickness of the uterus treated. The whole uterine wall is included as it becomes difficult to discriminate between exophytic and infiltrative disease without histopathologic specimens obtained by surgical removal of the uterus [12]. Some of the common methods of intra-uterine irradiation include single tandem with ovoids, double tandem, or Heyman capsule placement. The goal of this study was to evaluate an alternative novel method of brachytherapy using an intra-uterine inflatable balloon to irradiate the uterine wall. A dosimetric analysis of four different brachytherapy techniques was performed: single tandem, double tandem, Heyman capsules, and inflatable balloon to evaluate target coverage and dose homogeneity.

\section{Methods and Materials}

Four women with primary early stage endometrial cancer deemed to be unresectable participated in the study. Informed consent was obtained from each patient for each applicator used based on institutional guidelines. The single and double tandems used were standard Fletcher-type (Nucletron Corporation, Columbia, MD). Heyman capsules were the disposable after loading type (Radiation Products Design, Inc., Albertville, MN). A total of 8 capsules were used. The inflatable balloon with a central bi-lumen catheter is the Mammo Site Radiation Therapy System (Proxima Therapeutics, Alpharetta, GA) that is currently used for local breast irradiation. Forty cubic centimeter volume was utilized. Instrument insertion was performed in an operative suite under conscious sedation. Following placement of the brachytherapy instruments, patients were then transported to the CT simulator. Rectal and bladder contrast was utilized for target delineation following which CT images were obtained on the pelvis. CT images were then transported to the planning software [13].

Treatment planning and dosimetric analyses were done with HDR PLATO Brachytherapy (v14.2.3) software (Nucletron Corporation). Plan optimization was performed using three-dimensional (3-D) interpolated contours of the target volume (uterus) based on CT data. Homogeneity (dose at inner surface/dose at outer surface of the uterine wall) and conformality indices were then calculated for each apparatus. Figures 1-4 illustrate the sample isodose lines generated by the different applicators.

\section{Results}

The average dose gradient within the target (uterine wall) is highest with the tandem methods, and closely followed 


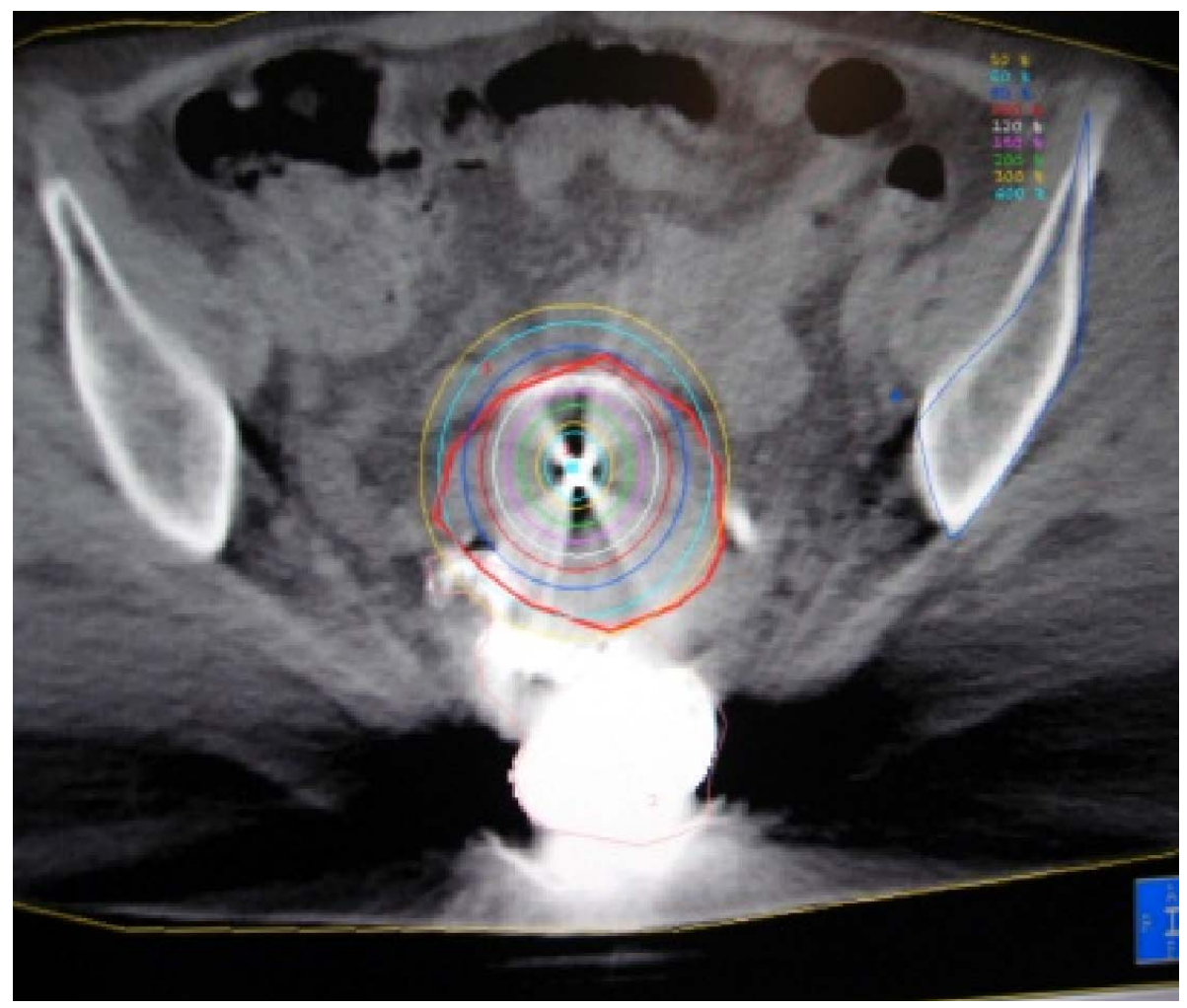

Figure 1. Isodose with single tandem.

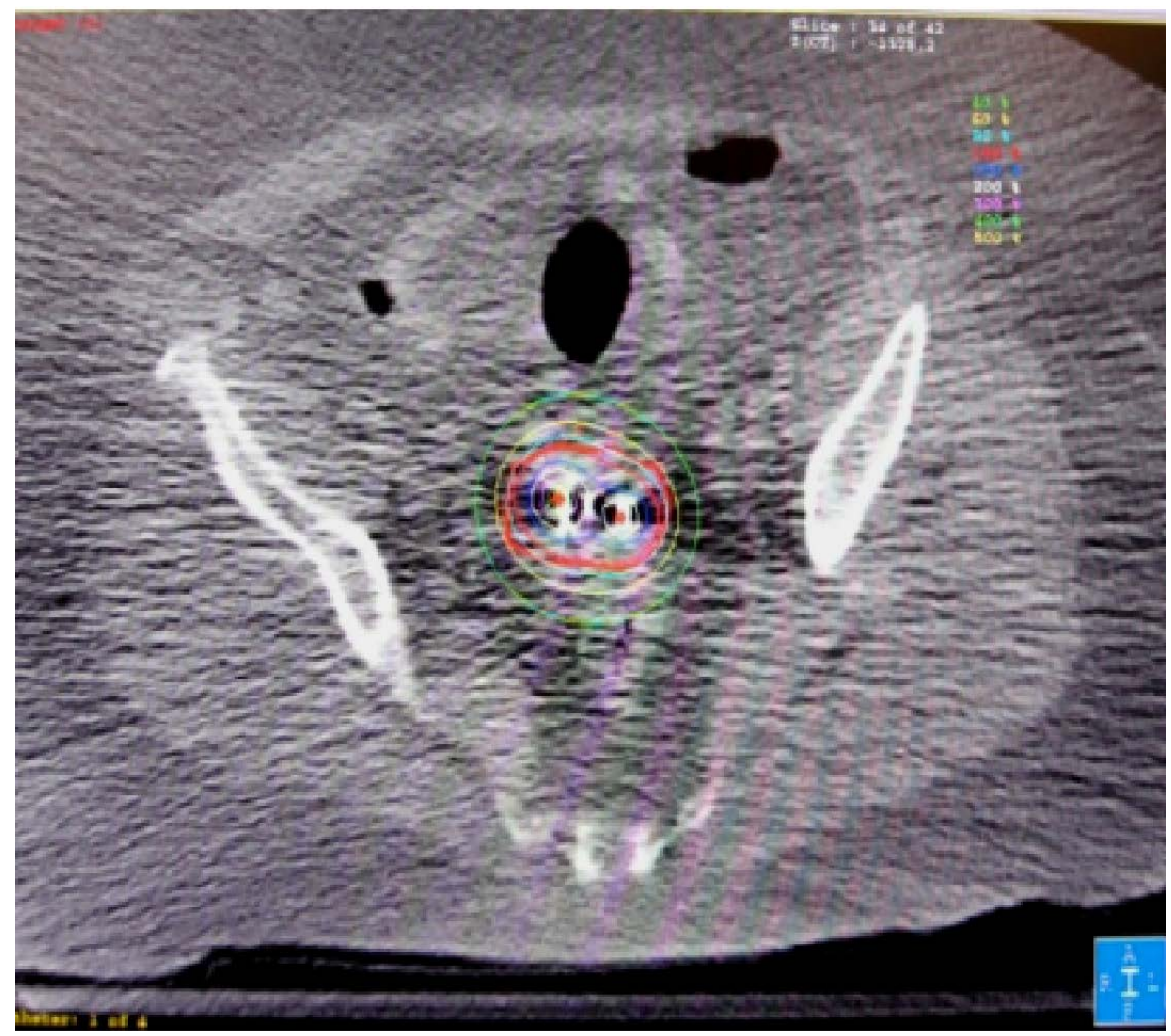

Figure 2. Isodose with double tandem. 


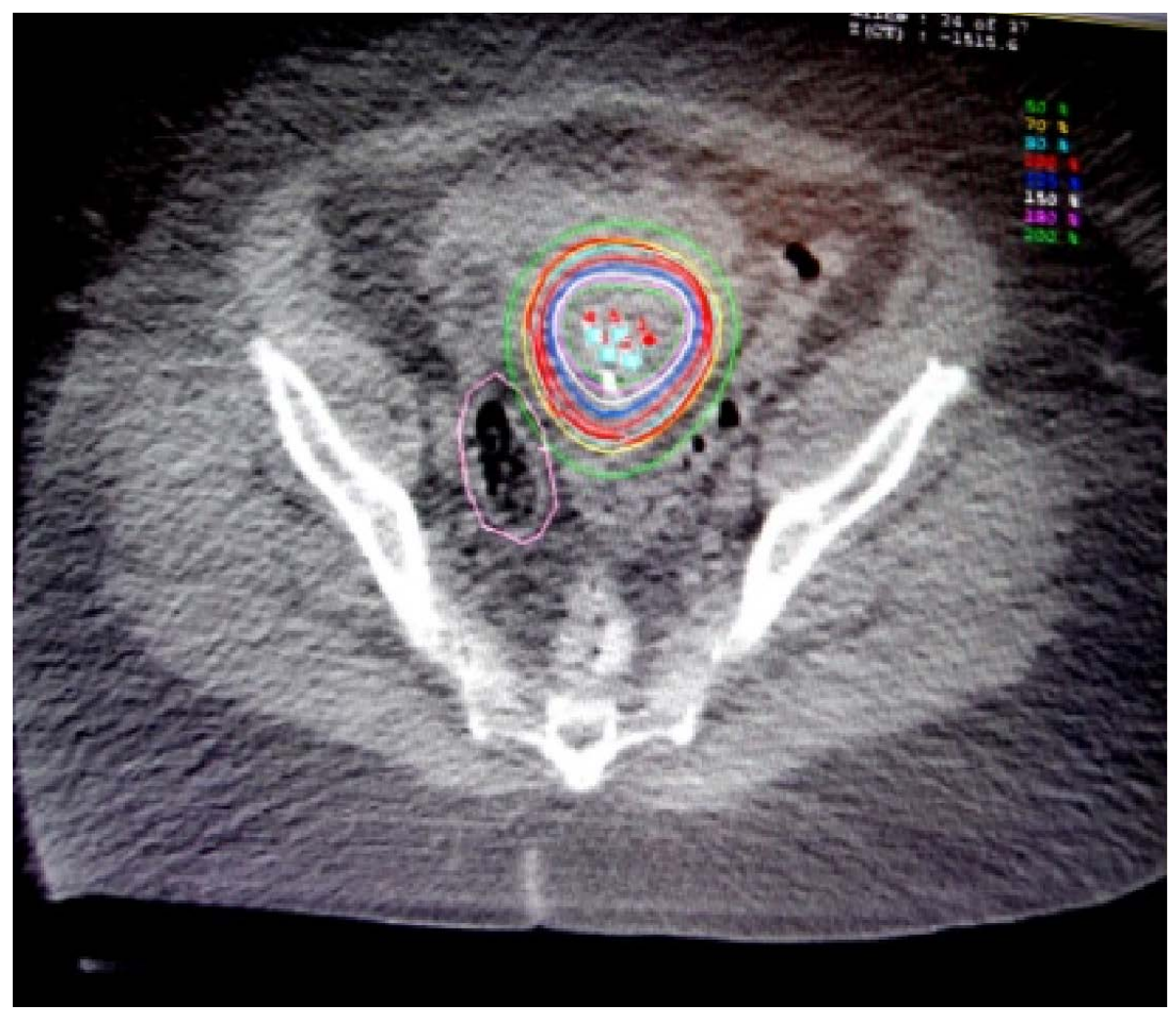

Figure 3. Isodose with Heyman capsules.

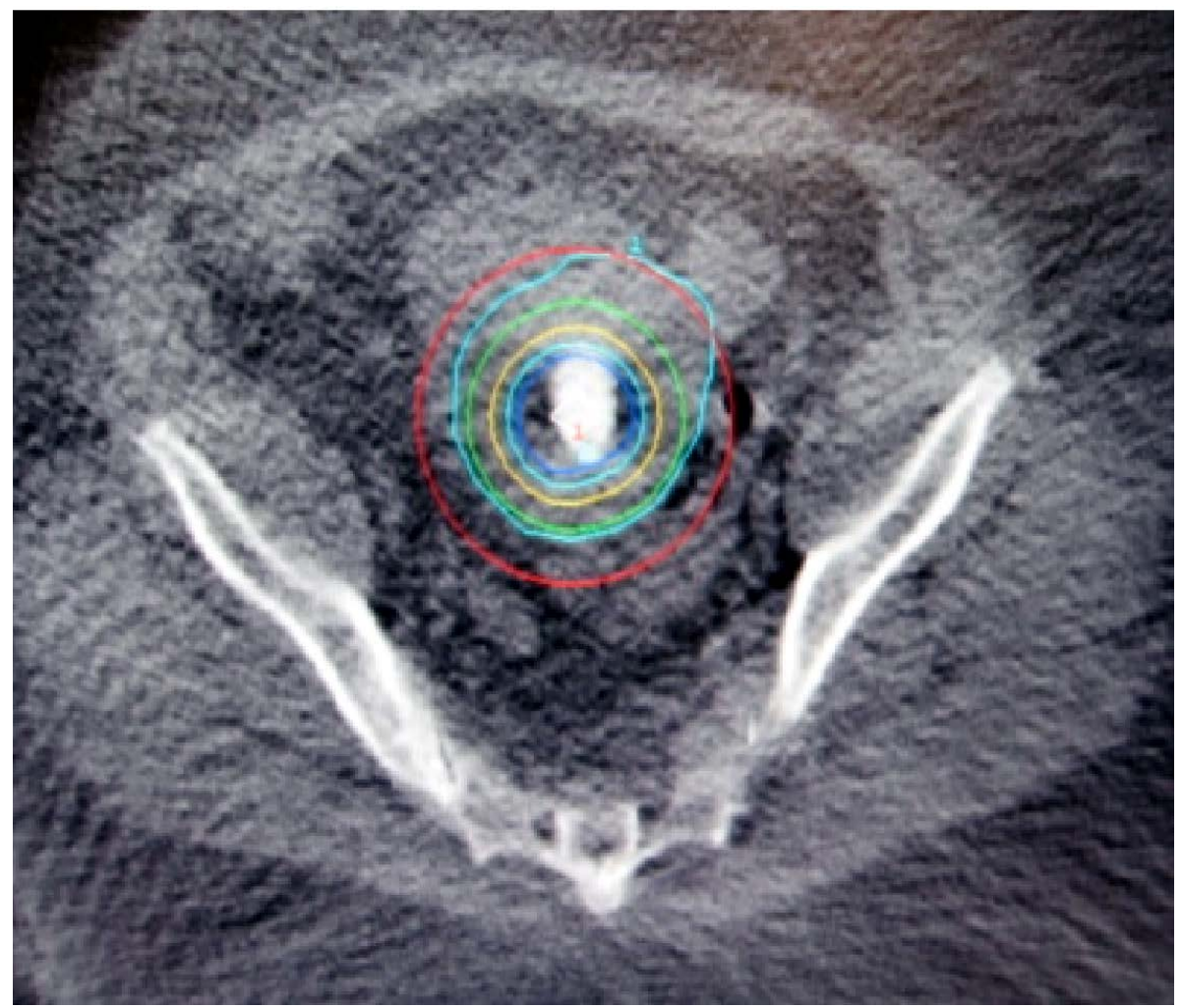

Figure 4. Isodose with inflatable balloon. 
by Heyman capsules. The balloon method showed the least dose gradient across the uterine wall. The corresponding average homogeneity indices were 3.81, 3.83, 2.97, 2.50 for single tandem, double tandem, Heyman capsules, and Balloon method respectively. With proper optimization, the 3-D dose clouds of Heyman capsules and balloon catheter method conformed to the shape of each uterus better than the single or double-tandem methods, especially in the anterior-posterior (AP) direction as suggested by the conformality indices (1.16 and 1.20 for the Heyman capsules and inflatable balloon respectively compared to 2.33 and 1.32 for the tandem techniques). The results are summarized in Table 1 . The intra-uterine balloon and single tandem were noted by the practicing radiation physician to have the easiest and fastest placement compared to the double-tandem and Heyman capsule placement, with Heyman capsule placement being the most technically challenging.

\section{Discussion}

Women who present with inoperable endometrial cancer are often poor surgical candidates because of other medical problems. These patients can be treated effectively with definitive radiation therapy based on outcomes from multiple retrospective series [2]-[11]. However, the very problem that deems them inoperable can also make them high risk for radiation treatment related complications when considering which radiation treatment technique to use. These patients can be treated with a combination of therapies including external beam and intracavitary brachytherapy. Whichever treatment is chosen, the ultimate goals are to provide adequate dose to treat the disease, while at the same time, sparing dose to normal structures in the pelvis.

Standard intracavitary brachytherapy techniques have included single tandem, double tandem and Heyman capsules for patients treated with intact uterus [12]. Each technique has its advantages and disadvantages which can significantly affect confidence of the user. Most of these methods can be placed under conscious sedation, obviating the need for general anesthesia, which may have precluded surgery in the first place.

In this study, a novel new technique has been proposed using an intra-uterine inflatable balloon similar to those used in other cancer sites to provide adequate dose coverage. Inflatable balloon techniques have been used in breast cancer as a means to deliver partial breast irradiation with acceptable local control [14].

Based on this dosimetric analysis, the intra-uterine inflatable balloon appears to have the best dosimetric advantages for the treatment of the uterine wall compared to single tandem, double tandem and Heyman capsules. Better dose homogeneity and conformality was found for the intra-uterine balloon technique. Improved homogeneity can lead to better control rates as the entire target volume receives an adequate tumoricidal dose. Better conformality can reduce high doses to critical organs by providing rapid dose fall and thus reduce acute and late toxicities. The intra-uterine balloon was noted to be the easiest and fastest to place compared to Heyman capsules and double tandems. The intra-uterine single tandem and Heyman capsules were also noted to have risk of slippage or displacement compared to the double tandem and inflatable balloon which are secured by intra-uterine anchoring.

Recent evidence has been published using an intra-vaginal balloon technique (GynSite ${ }^{\circledR}$ ) in patients requiring adjuvant radiation for endometrial cancer. Miller et al. treated 30 consecutive operable patients with endometrial cancer with 6 weekly fractions of adjuvant HDR brachytherapy using the intra-vaginal balloon. They reported excellent radiation dose coverage with acceptable doses to the bladder and rectum [15]. While this study pertains to patients who initially received surgical resection, the acceptable coverage using an intra-vaginal balloon provides more evidence for this dosimetric analysis using an intra-uterine balloon in inoperable patients.

There are limitations of the data presented due to anatomical differences in the shape, thickness, and size of the uterus of individual patients. CT artifacts may have obscured anatomy. Furthermore, the selection of the isodose lines at high dose are estimates and calculations of homogeneity and conformality indices based on these numbers and DVH's can be potentially misleading. However, the observed trend of better coverage with more homogenous dose across the uterine wall using the intrauterine balloon is encouraging and warrants further

Table 1. Average homogeneity and conformality indices for tandem, Heyman capsules, and inflatable balloon techniques.

\begin{tabular}{ccccc}
\hline Indices & Single Tandem & Double Tandem & Heyman Capsules & Inflatable Balloon \\
\hline Homogeneity & 3.81 & 3.83 & 2.97 & 2.50 \\
Conformality & 2.33 & 1.32 & 1.16 & 1.20 \\
\hline
\end{tabular}

Homogeneity index $=$ dose at inner surface/dose at outer surface of the uterine wall. Conformality index $=$ target volume/treatment volume. 
investigation. The next step of investigation would then be to proceed with a Phase I/II study to determine clinical outcomes, as this study looked at dosimetric outcome as its primary endpoint.

\section{Conclusion}

The intra-uterine inflatable balloon appears to have the best overall dosimetric advantages for the treatment of uterine wall in inoperable patients with uterine cancer. Furthermore, the potential ease of use, shorter time of applicator placement, and potentially superior patient comfort, warrant further investigation and subsequent clinical implementation.

\section{References}

[1] American Cancer Society (2013) Endometrial (Uterine) Cancer. http://www.cancer.org/Cancer/EndometrialCancer/DetailedGuide/endometrial-uterine-cancer-key-statistics

[2] Nguyen, T.V. and Petereit, D.G. (1998) High-Dose-Rate Brachytherapy for Medically Inoperable Stage I Endometrial Cancer. Gynecologic Oncology, 71, 196-203. http://dx.doi.org/10.1006/gyno.1998.5148

[3] Fishman, D.A., Roberts, K.B., Chambers, J.T., et al. (1996) Radiation Therapy as Exclusive Treatment for Medically Inoperable Patients with Stage I and II Endometrioid Carcinoma with Endometrium. Gynecologic Oncology, 61, 189196. http://dx.doi.org/10.1006/gyno.1996.0123

[4] Rouanet, P., Dubois, J.B., Gely, S., et al. (1993) Exclusive Radiation Therapy in Endometrial Carcinoma. International Journal of Radiation Oncology*Biology*Physics, 26, 223-228.

[5] Lehoczky, O., Bosze, P., Ungar, L., et al. (1991) Stage I Endometrial Carcinoma: Treatment of Nonoperable Patients with Intracavitary Radiation Therapy Alone. Gynecologic Oncology, 43, 211-216. http://dx.doi.org/10.1016/0090-8258(91)90022-W

[6] Kucera, H., Knocke, T.H., Kucera, E., et al. (1998) Treatment of Endometrial Carcinoma with High-Dose-Rate Brachytherapy Alone in Medially Inoperable Stage I Patients. Acta Obstetricia et Gynecologica Scandinavica, 77, 10081012. http://dx.doi.org/10.1080/j.1600-0412.1998.771011.x

[7] Knocke, T.H., Kucera, H., Weidinger, B., et al. (1997) Primary Treatment of Endometrial Carcinoma with HighdoseRate Brachytherapy: Results of 12 Years of Experience with 289 Patients. International Journal of Radiation Oncology*Biology*Physics, 37, 359-365.

[8] Kupelian, P.A., Eifel, P. and Tornos, C. (1993) Treatment of Endometrial Carcinoma with Radiation Therapy Alone. International Journal of Radiation Oncology*Biology*Physics, 27, 817-824.

[9] Grisby, P.W., Kuske, R.R., Perez, C.A., et al. (1987) Medically Inoperable Stage I Adenocarcinoma of the Endometrium Treated with Radiotherapy Alone. International Journal of Radiation Oncology*Biology*Physics, 13, 483-488.

[10] Niazi, T.M., Souhami, L., Portelance, L., et al. (2005) Long-Term Results of High-Dose-Rate Brachytherapy in the Primary Treatment of Medically Inoperable Stage I-II Endometrial Carcinoma. International Journal of Radiation Oncology*Biology*Physics, 63, 1108-1113.

[11] Coon, D., Beriwal, S., Heron, D.E., et al. (2008) High-Dose-Rate Rotte "Y” Applicator Brachytherapy for Definitive Treatment of Medically Inoperable Endometrial Cancer: 10-Year Results. International Journal of Radiation Oncology*Biology*Physics, 71, 799-783.

[12] Potter, R., Gerbaulet, A. and Haie-Meder, C. (2002) The GEC-ESTRO Handbook of Brachytherapy. Endometrial Cancer. http://estro-education.org/publications/Documents/IB\%2015\%2029072002\%20Endometrial\%20Cancer\%20print_proc TW.pdf

[13] Nag, S., Erickson, B., Parikh, S., et al. (2000) The American Brachytherapy Society Recommendations for High-DoseRate Brachytherapy for Carcinoma of the Endometrium. International Journal of Radiation Oncology*Biology*Physics, 48, 779-790.

[14] Vicini, F., Beitsch, P., Quiet, C., et al. (2011) Five-Year Analysis of Treatment Efficacy and Cosmesis by the American Society of Breast Surgeons Mammo Site Breast Brachytherapy Registry Trial in Patients Treated with Accelerated Partial Breast Irradiation. International Journal of Radiation Oncology*Biology*Physics, 79, 808-817.

[15] Miller, D.A., Richardson, S. and Grigsby, P.W. (2010) A New Method of Anatomically Conformal Vaginal Cuff HDR Brachytherapy. Gynecologic Oncology, 116, 413-418. http://dx.doi.org/10.1016/j.ygyno.2009.10.044 
Scientific Research Publishing (SCIRP) is one of the largest Open Access journal publishers. It is currently publishing more than 200 open access, online, peer-reviewed journals covering a wide range of academic disciplines. SCIRP serves the worldwide academic communities and contributes to the progress and application of science with its publication.

Other selected journals from SCIRP are listed as below. Submit your manuscript to us via either submit@scirp.org or Online Submission Portal.
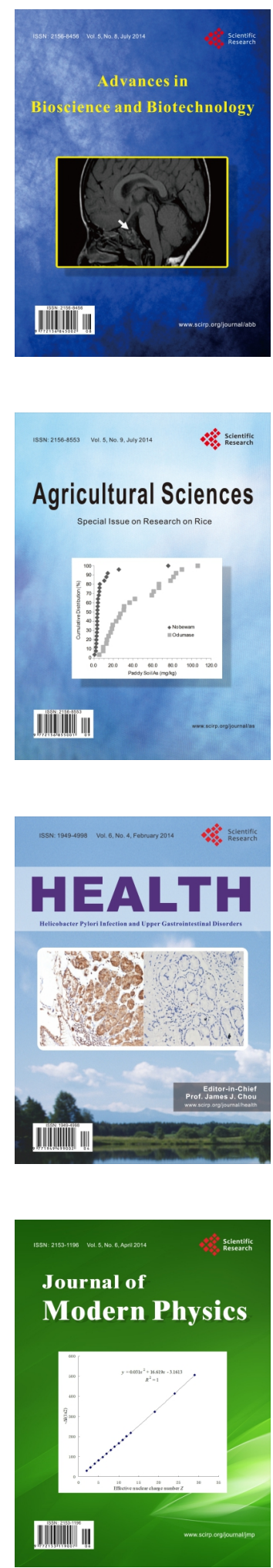
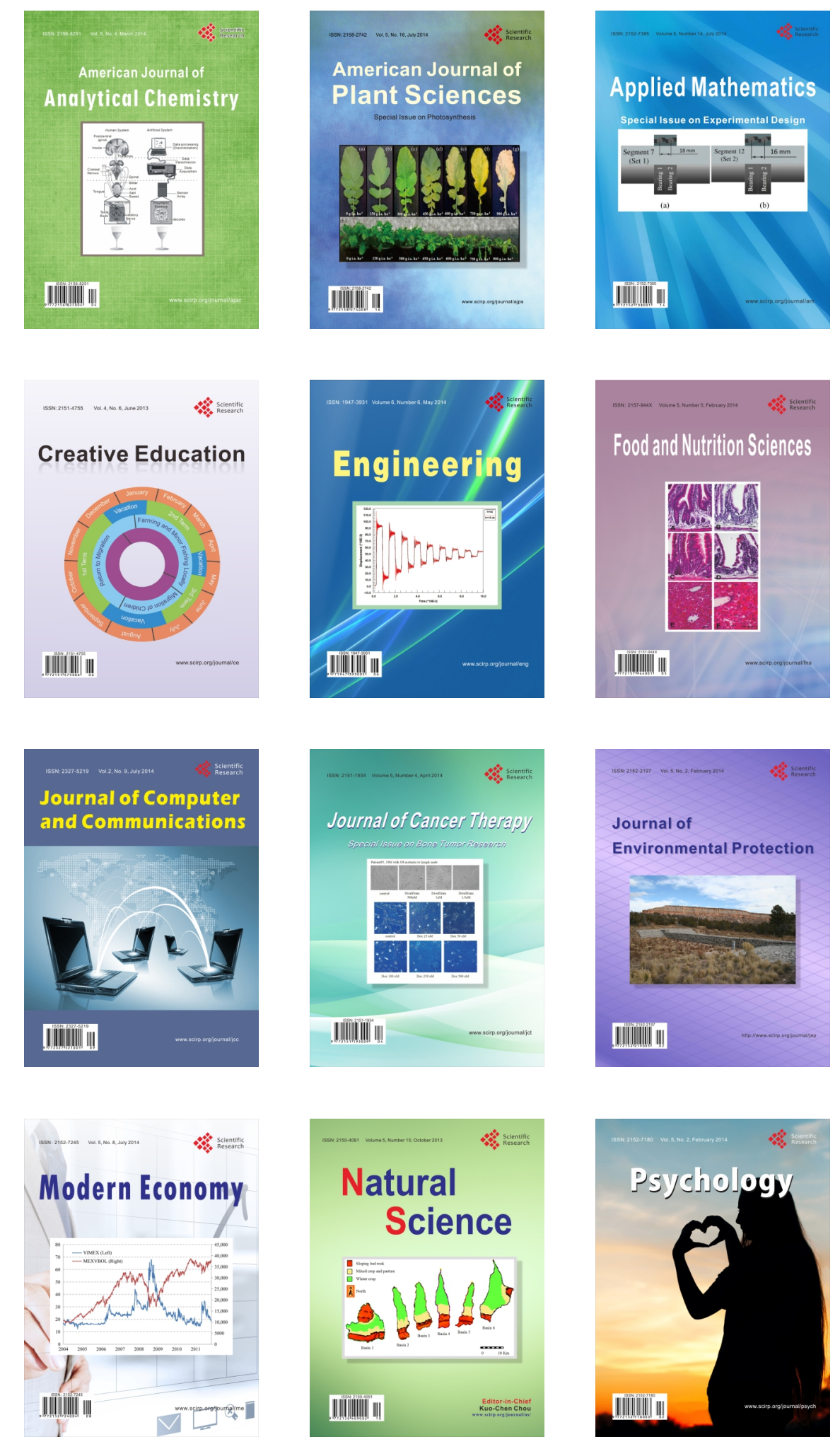